DOI: $10.12737 / \mathrm{jllcl} .2021 .003$

\title{
Concilier la défense des identités nationales et le respect des engagements internationaux : libres propos sur un défi à relever*
}

\section{Bertrand Mathieu, Professeur agrégé des Facultés de droit, Membre étranger de l'Académie des sciences de Russie}

Le sujet est d'une brûlante actualité et présente un caractère quasiment universel. D'une part le phénomène de mondialisation, ou d'internationalisation, dépasse largement le cadre économique et financier et touche également aux valeurs qui sont celles des nations en construisant peu à peu un système à vocation universelle qui ne l'est pas vraiment mais qui aspire à le devenir. Il en est ainsi, par exemple, d'une conception essentiellement individualiste des droits fondamentaux, de la réécriture de l'histoire à la lumière de conceptions contemporaines et anachroniques. De la même manière, de manière plus indirecte mais plus profonde, les GAFA tendent à uniformiser les modes de pensée, tout en créant et développant des communautés qui s'organisent autour de leurs propres systèmes de valeurs.

Si l'on se recentre sur le terrain juridique, le droit international, ou régional, largement construit par des juges supranationaux et relayé par les juges nationaux conduit à marche forcée vers l'uniformité.

Ces phénomènes contribuent à remettre en cause les identités nationales voire à les dévitaliser. Mais, d'un autre côté, cette tentative d'uniformisation, conduit les peuples et certains états à opérer un mouvement de repli vers une défense de leur identité nationale.

Le défi auquel, nous juristes, sommes confrontés et d'articuler les exigences résultant de ce mouvement d'internationalisation, auquel les États ont adhéré au moyen de dispositions conventionnelles, et la protection de l'identité nationale qui justifie l'existence même de l'État.

Dans les analyses qui suivent, je m'inscrirai essentiellement dans le cadre des rapports entre le Conseil de l'Europe et les États membres. Les propositions qui sont faites, sont succinctes, elles méritent débats et approfondissements, elles visent à dépasser un constat de conflit potentiel pour tenter de trouver des mécanismes de résolution de ces conflits.

Les lignes directrices que je retiendrai sont les suivantes : D'une part, il convient de veiller à ce que les Etats ne se voient pas imposer des contraintes auxquelles ils n'ont pas librement adhéré et qu'ils conservent leur libre arbitre concernant ce qui relève de leur identité nationale ; d'autre part les Etats doivent être soumis au respect des engagements qu'ils ont pris. Plus concrètement cela implique, au niveau européen, de maintenir les mécanismes de respect des traités notamment la juridiction de la Cour européenne des droits de l'homme, mais aussi de délimiter le plus clairement possible ce qui relève des identités nationales par exemple les questions relatives à la conception de la famille ou de la religion l'accès à son territoire, et ce qui relève de valeurs communes, par exemple, une justice indépendante le respect des droits de la défense la dignité humaine ou le respect d'élections libre.

C'est au constituant de fixer les valeurs identitaires et au juge national de les faire respecter, c'est au traité de fixer les valeurs communes et aux juges européens de les faire respecter. La question est alors bien évidemment de savoir comment articuler la protection de ces deux identités.

Le problème tient au fait que les rapports entre le droit international et le droit constitutionnel national ne se prêtent pas à une hiérarchisation unique des normes, ce qui conduirait à la création d'un système fédéral constitutionnel. On relèvera incidemment que la Cour européenne des droits de l'homme s'est inscrite dans cette logique en se définissant comme une cour constitutionnelle.

En réalité la situation actuelle se traduit par l'existence de plusieurs ordres juridiques : international, européen, national, dont les rapports sont essentiellement régulés par les juges, ce qui les conduit à intervenir largement dans les compétences des organes politiques.

Admettre la supériorité inconditionnelle de la jurisprudence constructive de la Cour européenne des droits de l'homme ou, au contraire, la prévalence, tout aussi inconditionnelle des règles constitutionnelles nationales, ne peut conduire qu'à une impasse ou à un blocage.

I. Identité européenne v. identités nationales : une relation dialectique

On partira d'une rapide analyse de la manière dont les systèmes de valeurs traduisant ces identités sont établis tant dans le système du Conseil de l'Europe que dans les systèmes nationaux.

\section{Le Conseil de l'Europe et le dessein d'une identité} européenne

S'agissant du Conseil de l'Europe, l'organe central de cette organisation est le Comité des ministres composé des ministres des affaires étrangères des États parties. Mais en matière de droits fondamentaux le rôle majeur est occupé par la Cour EDH. Le Conseil de l'Europe a créé une multitude d'organes dont le rôle est essentiellement consultatif et qui vont, dans leurs domaines de spécialités, participer à sa mission. Tel est le cas de la Commission européenne pour la démocratie et le droit dite « Commission de Venise ».

Ainsi, les États européens se trouvent véritablement encadrés par une multitude d'organes compétents pour

* Intervention de Bertrand Mathieu au Xème Congrès international de Droit comparé «Évolution constitutionnelle en Russie et dans le monde moderne : dialectique de l'universel et du national », Moscou 4 décembre 2020 (visioconférence). 
s'assurer du respect des valeurs européennes. L'action combinée de ces organes tisse un maillage efficace en faveur de la protection et de la promotion des droits fondamentaux et des valeurs européennes. Dans ce schéma, la Cour européenne des droits de l'Homme tend à se considérer, comme un juge néo-constitutionnel de l'Europe. Elle assure en quelque sorte, une transition entre soft law et hard law par une sanction juridictionnelle.

En ce sens, la Cour EDH se reconnaît le droit d'assurer à la fois l'identification des valeurs européennes et la ligne de partage entre ces valeurs européennes et la marge de manœuvre laissée aux États. Elle estime qu'il lui appartient à la fois d'adapter les droits reconnus par la Convention à ce qu'elle estime être les évolutions de la société européenne, ce qui peut la conduire, le cas échéant, à reconnaître des droits non-inscrits dans la Convention. Par ailleurs, La Cour considère qu'elle doit prendre en compte toute règle pertinente du droit international applicable aux relations entre les parties contractantes pour interpréter les droits et libertés reconnus par la Convention, qui n'est plus l'unique cadre de référence. Enfin, la Cour interprète librement le principe de subsidiarité au regard, notamment, des évolutions législatives des États membres -d'une majorité d'entre eux, de la quasi-totalité d'entre eux ? — modifiant ainsi l'esprit de la Convention dont la substance se modifie au regard de la prise en compte de l'évolution des droits nationaux.

La légitimité des organes de caractère intergouvernemental est essentiellement politique. La légitimité juridique des juges européens est encore d'une autre nature. Il convient également de s'interroger sur la légitimité des comités d'experts.

De ce dernier point de vue, la Commission de Venise a essentiellement une mission idéologique qui consiste à diffuser les « valeurs fondamentales de l'État de droit, des droits de l'homme et de la démocratie ». C'est en fait ce dernier triptyque qui, pour la Commission, définit sa compétence. Il s'agit d'une extension considérable des compétences du Conseil de l'Europe fondées essentiellement sur les objectifs du Conseil de l'Europe tels qu'ils sont rappelés dans le Préambule de son statut (Traité de Londres du 5 mai 1949). Ainsi la référence à la démocratie tient au fait que ce terme figure dans ce Préambule. Sur ce fondement la compétence de la Commission s'étend à l'organisation institutionnelle et politique des États. L'article $1-2$ du statut précise que les compétences de la Commission consistent, notamment, à examiner les principes et les techniques constitutionnels, législatifs et administratifs, les droits et libertés fondamentaux le droit des collectivités locales. Par ailleurs, si ses pouvoirs sont uniquement consultatifs, la réalisation proprio motu d'études générales, lui permet, de fait, de constituer son propre corpus de normes de références.

Cette action est bénéfique en ce qu'elle permet de déterminer le corpus des valeurs communes européennes.
Sa fonction peut s'étendre au-delà du respect de ces valeurs à partir du moment où elle peut, notamment, jouer un rôle de Conseil vis-à-vis des États dans le cadre de la construction et de l'évolution de leurs systèmes politiques. Mais la légitimité de cette institution tient à sa fonction de conseil et d'expertise. En revanche, plus discutable est le fait que certains de ses avis peuvent se voir conférer une portée contraignante par le truchement de la jurisprudence de la Cour EDH. Ainsi, par exemple, dans une affaire jugée par la Grande chambre le 27 avril $2010^{1}$, la Cour s'appuie largement sur un rapport de la Commission de Venise portant sur les modifications apportées au code électoral de la Moldavie en avril 2008. La Cour cite également un discours du Président de l'Assemblée parlementaire du Conseil de l'Europe qui encourageait vivement le Parlement moldave à obtenir pour la réforme envisagée l'aval de la Commission de Venise. Est alors cité au titre des « instruments pertinents $»$, le code de bonne conduite en matière électorale élaboré par la Commission de Venise ${ }^{2}$.

\section{Le constituant et la formulation de l'identité} nationale

Les valeurs qui structurent l'identité nationale ne peuvent être identifiées aux seuls droits fondamentaux. Leur prétention à l'universalisme, affaiblit le concept même d'identité nationale. En effet, ces droits fondamentaux sont très largement définis, ou interprétés, mais cela revient presque au même, par des structures supranationales de nature juridictionnelle, voire par des organisations non gouvernementales. Si l'on admet qu'un peuple se définit par son identité et que les droits de l'homme sont considérés comme ayant une portée universaliste, cette identité ne peut se dissoudre dans ces droits, alors même que ces droits peuvent faire partie de cette identité.

Le lien entre valeurs et identité tient au fait que la collectivité nationale n'est pas le fruit du hasard, ou un agrégat provisoire. Elle a ses racines dans le passé. «Elle constitue l'unique organe de conservation pour les trésors spirituels amassés par les morts, l'unique organe de transmission par lequel les morts puissent parler aux vivants $»^{3}$.

L'existence d'une communauté politique, condition première de la démocratie, implique la reconnaissance de son identité et donc une altérité par rapport à ce qui n'est pas elle.

Il convient d'abord de rechercher ce qui constitue l'identité d'une nation. Cette identité s'enferme difficilement dans une définition ou une énumération de critères juridiques. On peut cependant en trouver l'écho dans une Constitution : il en est ainsi, par exemple, de la langue, définie par Jacques Julliard comme « un signe de

\footnotetext{
${ }^{1}$ CEDH, 27 avril 2010, Tanase c/ Moldova, req. $n^{\circ} 7 / 08$.

${ }^{2} \mathrm{Cf}$. M. Guerrini, intervention à la conférence de Szeged dans le cadre du projet Balaton, 2014.

${ }^{3}$ S. Weil, L'enracinement, 1949, Folio, 2016, p. 16.
} 
ralliement, une culture, un esprit, une forme de rapport au monde $»^{4}$. C'est bien sûr, un territoire, une géographie. C'est aussi une culture, une littérature, une architecture. C'est une spiritualité, une religion.

Si cette question de l'identité nationale est difficilement appréhendée par le droit, le besoin de son affirmation juridique, face à un ordre juridique qui tend à être exclusivement construit (ou déconstruit) à partir des droits fondamentaux individuels, s'est manifestée, notamment, par deux constructions jurisprudentielles : celle des «principes inhérents à l'identité constitutionnelle » et celle de « l'ordre public immatériel $»^{5}$, c'est-à-dire à un ordre public bon contingent ne relevant pas de la sécurité, mais renvoyant à des valeurs.

\section{Comment concilier le respect de ces deux} identités ?

Les rapports de systèmes étant aujourd'hui essentiellement régulé par les juges et cette régulation conduisant par un effet d'induction sur les juridictions nationales à privilégier le rôle du juge européen, et donc de l'identité européenne au détriment des identités nationales, il convient de rechercher les conditions d'un nouvel équilibre.

1. Prévalence européenne et résistances nationales

En réalité, on peut observer que l'intégration européenne dans le système de l'Union européenne comme dans celui de la Convention européenne des droits de l'homme est l'œuvre de juridictions. Ces juridictions s'attribuent de fait, non seulement la mission de trancher des conflits, mais aussi une œuvre législative, voire constitutionnelle. On a pu parler en ce sens de fédéralisme juridictionnel.

Ainsi ce sont les juges qui régulent les rapports de système. C'est d'abord un facteur de renforcement de la puissance juridictionnelle face au pouvoir politique. Disposant d'un large éventail de normes juridiques, notamment européennes, elles même assez largement formulées par les juridictions européennes, le juge national y trouve une liberté plus qu'une contrainte. Il y trouve les outils pour assoir son pouvoir face à celui du politique.

L'inscription dans la Constitution russe de la suprématie des règles constitutionnelles sur celles issues de la jurisprudence de la Cour européenne des droits de l'homme, la résistance de certains États face à la politique migratoire de l'Union européenne, le recours à des mesures visant à encadrer le pouvoir des juges témoignent de résistances à la politique jurisprudentielle des cours européennes.

Face à cette construction, les pouvoirs politiques nationaux sont faibles et la protection des identités nationales qui relève de leur mission difficilement assurée. La crise qui affecte les identités nationales et leur rapport avec l'identité européenne est d'abord une crise du pouvoir politique.

\footnotetext{
${ }^{4}$ Le Figaro, 5 juin 2015.

${ }^{5}$ M. O. Peyroux-Sissoko, L'ordre public immatériel, Lextenso,
} 2018.
Enfin, il est difficile d'admettre que le juge européen soit, en dernier ressort, juge du caractère identitaire national. Sinon on s'inscrit dans le la logique d'un État fédéral dont la Cour européenne des droits de l'homme serait la cour suprême.

De ce conflit, qui n'est pas assumé et qui est mal résolu par des procédures juridictionnelles, rien de bon ne peut résulter. Soit l'on se dirigera vers un fédéralisme de fait, qui n'est pas assumé et qui à terme suscitera des révoltes de la part de citoyens qui ne sont plus que spectateurs, soit par un éclatement des structures européennes du fait du refus de certaines nations de se soumettre et d'abdiquer leur souveraineté.

\section{Propositions sommaires pour une résolution des} conflits

Dans le cadre limité de cette intervention, je voudrais seulement lancer quelques pistes, qui sont à la fois très partielles et beaucoup trop schématiques.

Redéfinir l'articulation des compétences nationales et des compétences européennes

Cette définition doit être l'œuvre des politiques. De ce point de vue, et à la décharge des juges, il convient de relever que le développement du pouvoir juridictionnel s'inscrit dans le contexte d'un refus des responsables politiques d'exercer leur pouvoir ou de leur incapacité à s'entendre.

Il s'agit en fait de déterminer clairement ce qui doit relever des compétences confiées à des structures, européennes, et les compétences et pouvoirs qui doivent rester entre les mains des États. Pour ce faire, il convient de distinguer ce qui relève de l'identité européenne, qui justifie l'association d'un certain nombre d'États, et ce qui relève de l'identité nationale.

La réflexion doit s'engager dans deux directions. Définir plus précisément les compétences nationales et les compétences européennes. Il s'agit en fait d'engager une réflexion sur ce que les États entendent réellement mettre en commun.

Il convient alors d'admettre que l'affirmation d'un principe identitaire constitue une réserve à une absolue prévalence des ordres européens sur l'ordre national, prévalence qui est fixée par les traités et ne vaut que parce qu'elle est acceptée par les Constitutions nationales.

Faire respecter le principe de subsidiarité

Les grandes lignes de cette répartition des compétences, établie à la suite d'un travail essentiellement de nature politique, il sera alors plus facile de faire respecter par la Cour européenne des droits de l'homme le principe de subsidiarité.

Ce principe implique que ce n'est que si la protection constitutionnelle s'avère insuffisante que la question doit être traitée au niveau européen. En effet, comme le relève Jean Paul Costa, ce principe implique que la tâche d'assurer le respect des droits consacrés par la Convention européenne incombe en premier lieu aux autorités des États contractants et non à la Cour, cette dernière n'intervenant qu'en cas de défaillance des 
autorités nationales. Ainsi, s'agissant de droits ou libertés appartenant à la fois au corpus constitutionnel et au corpus conventionnel, il convient de considérer que cette protection est d'abord assurée, s'agissant du contrôle de la loi, dans l'ordre constitutionnel.

Aujourd'hui la Cour semble évoluer en faveur de la reconnaissance d'un principe de subsidiarité sur certaines questions dites « de société $»^{6}$ les laissant à l'appréciation du législateur national. Mais l'appréciation de la portée de ce principe reste entre ses mains

Passer d'une obligation de soumission à une obligation de dialogue constructif

Un conflit du type de celui qui a opposé la cour constitutionnelle allemande à la Cour de justice de l'Union européenne, ou pour rester dans le cadre du Conseil de l'Europe, la résistance de la Grande-Bretagne à la jurisprudence de la Cour européenne des droits de l'homme relative aux droits de vote des détenus, témoignent à la fois de l'impasse que constitue l'exigence d'un seul rapport vertical entre juridictions européennes et juridictions nationales et de la nécessité de trouver un mode de résolution des conflits. On peut ainsi imaginer que s'agissant des rapports entre juridictions

${ }^{6}$ Par exemple, en matière de filiation, CEDH 22 mars 2012, $\mathrm{n}^{\circ}$ 45071/09 Ahrens c Allemagne et $n^{\circ} 23338 / 09$ Kautzor c Allemagne. les juridictions nationales puissent réinterroger les juridictions européennes lors qu'un conflit se produit ou est susceptible de naître. On pourrait également imaginer la création d'une sorte de Tribunal des conflits composé pour les affaires qui posent réellement problème de juges nationaux et de juges européens afin d'arbitrer les conflits de compétence, comme cela existe en France s'agissant des conflits de compétence entre le juge judiciaire et le juge administratif. Il ne s'agirait pas de créer un nouveau niveau de juridiction et de complexifier encore une organisation déjà lourde, mais de créer un organe de conciliation au fonctionnement souple. Par exemple, s'agissant d'un conflit particulier entre la Cour EDH et une juridiction constitutionnelle ou une juridiction suprême nationale, une formation ad 'hoc pourrait être réunie. Une formation plus pérenne pourrait être réunie pour traiter de questions récurrentes ou systémiques. En cas de nonrésolution des conflits, ou dans l'hypothèse la solution du conflit se heurterait, selon l'État concerné, à un principe fondamental reconnu par l'ordre constitutionnel, il conviendrait de rendre aux autorités politiques le pouvoir du dernier mot en la matière.

Un mot de conclusion : il convient de ne pas oublier que si la protection des droits et libertés fondamentales s'est épanouie dans le creuset européen, les États restent le cadre naturel de l'expression démocratique.

For citation. Mathieu B. Concilier la défense des identités nationales et le respect des engagements internationaux : libres propos sur un défi à relever. Journal of Foreign Legislation and Comparative Law, 2021, vol. 17, no. 1, pp. 15-18. DOI: 10.12737/jflcl.2021.003

Для цитирования. Матье Б. Concilier la défense des identités nationales et le respect des engagements internationaux : libres propos sur un défi à relever // Журнал зарубежного законодательства и сравнительного правоведения. 2021. T. 17. № 1. С. 1518. DOI: $10.12737 /$ jflcl.2021.003 